\title{
MAPEAMENTO DA SUSCETIBILIDADE A MOVIMENTOS DE MASSA NO MUNICÍPIO DE BELO HORIZONTE (MG) A PARTIR DA APLICAÇÃO DE ANÁLISE MULTICRITÉRIO
}

\author{
Dayane Caroline Freitas Carvalho $^{(\mathrm{a})}$, Cassio Dornas $^{(\mathrm{b})}$, Giovana Benvenuto $^{(\mathrm{c})}$
}

\author{
(a) Estudante de Mestrado em Análise e Modelagem de Sistemas Ambientais, Universidade Federal de Minas Gerais, \\ dayanecfc@gmail.com \\ (b) Estudante de Bacharelado em Geografia, Universidade Federal de Minas Gerais, cassiodornas@gmail.com \\ (c) Estudante de Bacharelado em Ciências Socioambientais, Universidade Federal de Minas Gerais, \\ giovana.bevenuto@gmail.com
}

\section{Eixo: GEOTECNOLOGIAS E MODELAGEM ESPACIAL EM GEOGRAFIA FÍSICA}

\begin{abstract}
Resumo
Os movimentos de massa estão entre os fenômenos naturais que mais provocam desastres naturais em encostas de climas tropicais úmidos. Diante disso, esta pesquisa objetivou desenvolver um mapeamento que indique a suscetibilidade aos movimentos de massa do município de Belo Horizonte (MG). Para tal, a metodologia consistiu em análises multicritério, onde foram atribuídos pesos as variáveis escolhidas de forma ponderada e, posteriormente, com a álgebra de mapas, realizou-se o cruzamento das variáveis e a geração de um mapa como produto.Como resultado foi elaborado o mapa de suscetibilidade a movimentos de massa, com áreas de baixa e alta suscetibilidade. Dados de ocorrência do fenômeno (2012-2016) foram adquiridos com a Defesa Civil de Belo Horizonte e quando plotados sobre o mapa concordaram de maneira eficaz sobre as áreas com alta suscetibilidade. Desta maneira, o produto gerado pode servir de base de dados para estudos mais detalhados e tomadas de decisão futuras.
\end{abstract}

Palavras chave: Movimentos de massa; mapeamento; multicritério.

\section{Introdução}

Atualmente os desastres naturais constituem um dos grandes problemas socioeconômicos do mundo, sendo que os fenômenos que mais se destacam são os terremotos, as inundações e os movimentos de massa (Guimarães, 2000). Segundo Wicander e James (2009), movimento gravitacional de massa é definido como sendo o movimento de descida, pela vertente abaixo, de material - solo e/ou rocha, sob a influência direta da gravidade, ou seja, os movimentos de massa ocorrem em vertentes com elevadas declividades, sob a influência do estresse gravitacional.

A região metropolitana de Belo Horizonte possui várias áreas de risco de movimentos de massa com elevado número de ocorrências. Além disso, existem casos de movimentações em áreas não consideradas de risco, cujas edificações possuem médio a elevado padrão construtivo, o que contribui para enfatizar que, além das atividades desordenadas de ocupação, os terrenos também apresentam susceptibilidade natural ao processo (Parizzi, et al, 2011). 
XVII Simpósio Brasileiro

de Geografia Fisica Aplicada

I Congresso Nacional

de Geografia Física
OS DESAFIOS DA GEOGRAFIA FÍSICA NA FRONTEIRA DO CONHECIMENTO

Instituto de Geociências - Unicamp

Campinas - SP

28 de Junho à 02 de Julho de 2017

Os movimentos de massa ocorrem por diferentes razões, entre elas, as condições naturais às quais o meio está condicionado, facilitando assim, a deflagração dos processos supracitados (VARGAS, 2015).

Deste modo, a busca por alternativas que possam contribuir com o diagnóstico dessas áreas vem ganhando espaço. Essa pesquisa integra técnicas de geoprocessamento para o desenvolvimento de análises conjuntas, envolvendo fatores físicos, sociais e econômicos.

Diante do exposto, o objetivo deste trabalho consiste em desenvolver um mapeamento que indique a suscetibilidade aos movimentos de massa do município de Belo Horizonte (MG), buscando identificar no município regiões mais frágeis e que necessitam de maior atenção, em relação as tomadas de decisões dos órgãos públicos.

\section{Caracterização da área}

O município de Belo Horizonte está localizado na região central do estado de Minas Gerais (Figura 1). Sua população estimada em 2016 era de 2.513 .451 pessoas, de acordo com os dados do IBGE. Sua área na unidade territorial é de aproximadamente $331,401 \mathrm{~km}^{2}$ e densidade demográfica de $7.167,00 \mathrm{hab} / \mathrm{km}^{2}$ de acordo com o Censo de 2010 (IBGE, 2010).

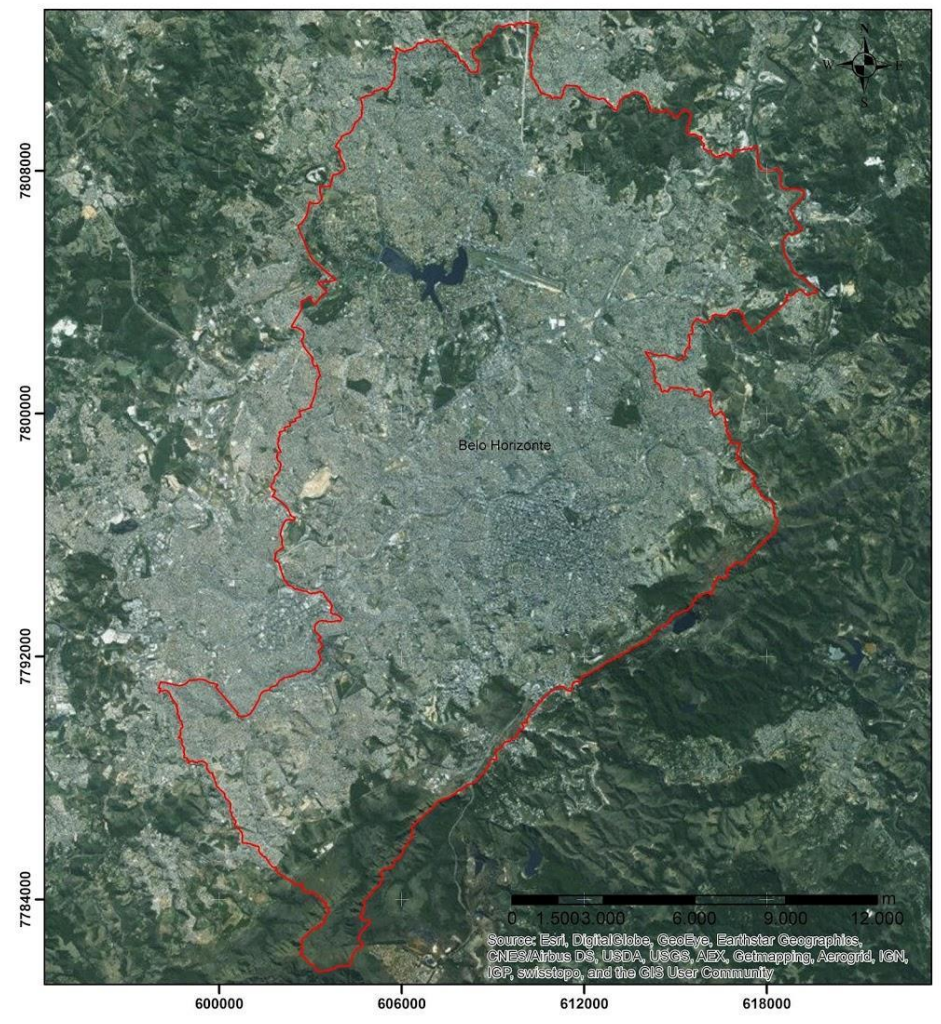

\section{Legenda}

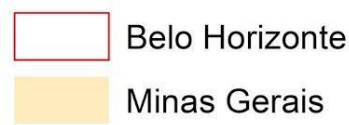

$1: 165.000$

Fonte: IGETEC/ERS

DATUM: Sirgas 2000

Coordenadas: UTM 235 
Figura 1 - Mapa de Localização da área de estudo

O município possui uma diversidade litológica e relevo acidentado, dispostos ao longo da serra do Curral, limite sul do município. Tal diversidade englobade sucessões de camadas rochosas de composições variadas: itabiritos, dolomitos, quartzitos, filitos e xistos diversos, de direção geral nordeste-sudeste e mergulho para o sudeste. (Prefeitura de Belo Horizonte, 2017).

Quanto à vegetação, o município é marcado por dois biomas principais: ocerrado e a mata atlântica. Devido à grande ocupação e acelerada urbanização de Belo Horizonte a maior parteda vegetação original foi substituída pela urbanização. Contudo, foram implementadas diversas áreas de preservação da vegetação pela cidade, sendo os mais conhecidos o Parque Serra do Curral, Parque das Mangabeiras, Parque Ecológico da Pampulha, Parque Municipal Américo Renné Giannetti, Serra da Moeda, entre outros.

\section{Metodologia}

O mapeamento das áreas susceptíveis amovimentos de massa cumpriuos procedimentos apresentados a seguir. Ao longo do desenvolvimento dessa pesquisa, foram realizadas pesquisas bibliográficas,com discussões sobre susceptibilidade, vulnerabilidade e risco; ocorrência de movimentos de massa no município; características físicas e ambientais do mesmo. Para a geração do mapa foram utilizadas as seguintes variáveis: curvas de nível (5 metros); mapa Geológico 1:50.000; densidade populacional; mancha urbana; cobertura vegetal; pluviosidade e ocorrências de movimento de massa 2012 a 2016.

Com a posse desses dados, foi aplicado o método de análise multicritério. Foram atribuídos pesos a cada variável utilizada, os quais foram definidos a partir da aplicação do modelo de AnalyticHierarchyprocess (AHP) para cada par de variáveis, considerando-se como a variável dependente o número de ocorrência de movimentos de massa e a independente como cada uma das variáveis de âmbito ambiental e socioeconômico. Os pesos de cada variável ficaram distribuídos da seguinte maneira: geologia (37,84\%); mancha urbana $(7,91 \%)$; pluviosidade $(9,96 \%)$; declividade $(36,25 \%)$ e densidade populacional $(8,02 \%)$.

Com os pesos de cada variável definidos, foram deliberados os valores das classes de cada critério seguindo a ordem crescente, da menos susceptível para mais suscetível. Em seguida, foi aplicado o método de álgebra de mapas para a construção da equação final, a qualresultou o mapa final.

Por fim, para a validação do mapa gerado foram utilizados os dados de ocorrência de movimentos de massa disponibilizados pela Defesa Civil de Belo Horizonte.Para a realização deste trabalho foram utilizadas somente as ocorrências classificadas como naturais, resultando num total de 1316 casos do ano 


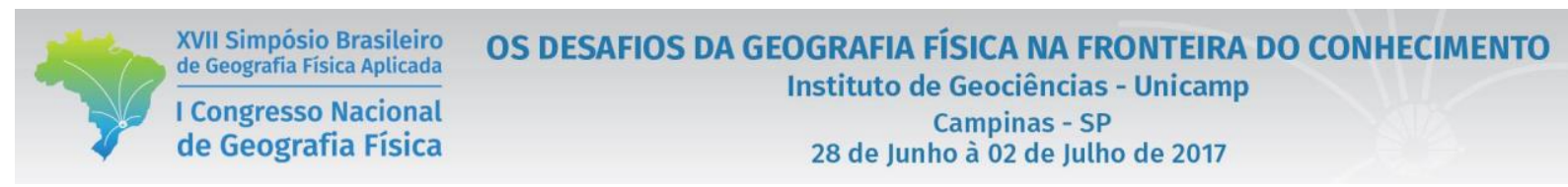

de 2012 a 2016.

\section{Resultados e Discussões}

Cada variável apresentada resultou em um mapa temático, sendo eles: litológico, declividade, ocupação, densidade populacional, pluviosidade e pontos de ocorrência de movimentos de massa. Com o cruzamento dos mapas citados anteriormente foi elaboradoo mapa de suscetibilidade a movimentos de massa em Belo Horizonte (figura 2).
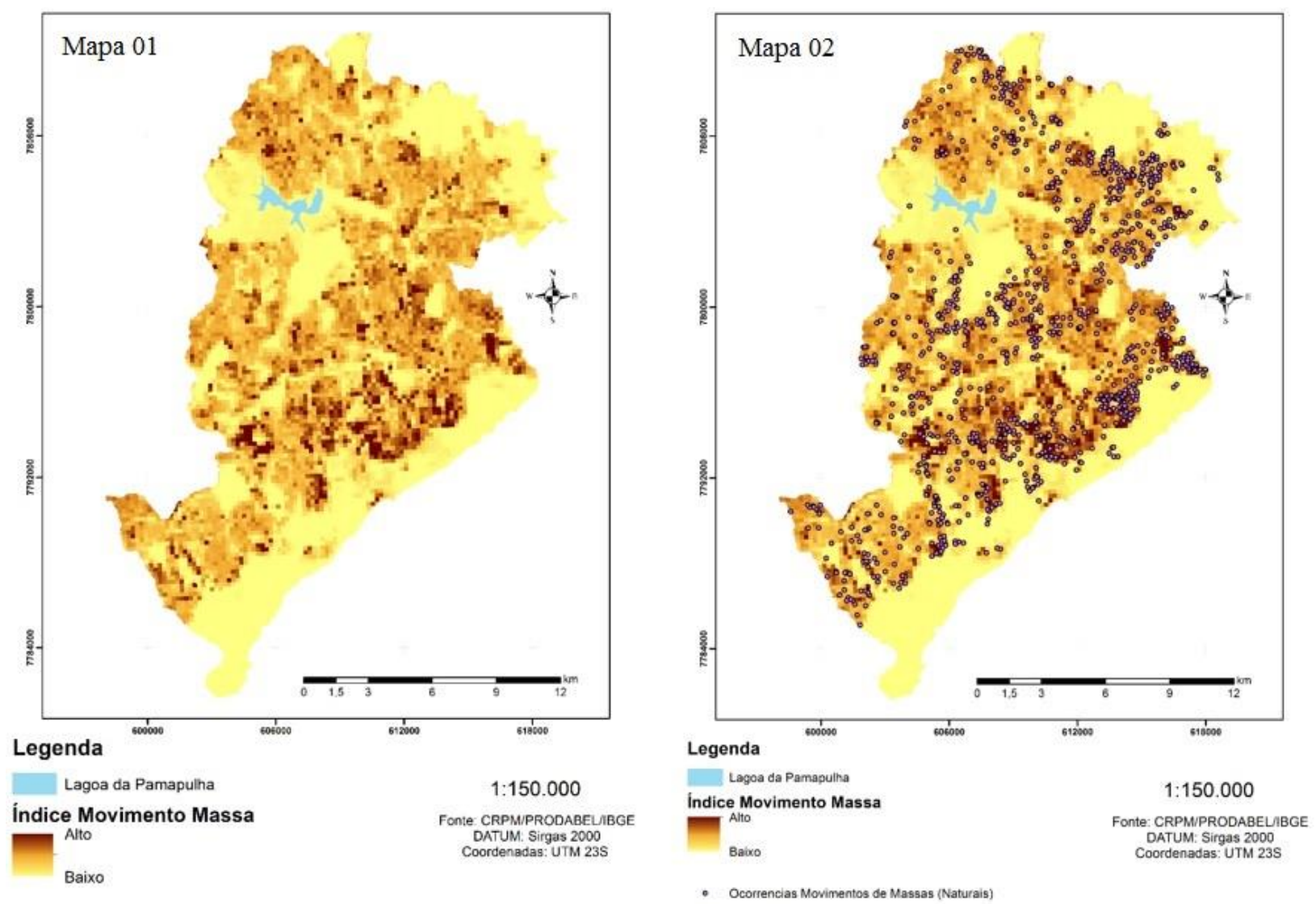

Figura 2 - Mapas de suscetibilidade a movimentos de massa. (Mapa 01 sem as ocorrências dos movimentos de massa e o Mapa 02 com as ocorrências de movimentos de massa (naturais) de 2012-2016).

Com os pontos plotados foi possível comparar os locais indicados pela análise multicritério com os dados de reais de ocorrências disponibilizados pela Defesa Civil e foi detectado uma concordância entre os dados, onde as regiões que mais apresentavam casos de ocorrência de movimentos de massa sobrepõem na maioria das vezes com as regiões que indicam alto índice de suscetibilidade. 


\section{Considerações Finais}

As ocorrências de movimentos massas em Belo Horizonte ocorrem com maior frequência em áreas de maior densidade de populacional.

Algumas regiões mesmo com altosvalores de declividade (variável com peso elevado) foram classificadas como áreas de baixa suscetibilidade, tal fenômeno acontece pela compensação realizada pelas outras variáveis, como baixa densidade populacional e predominância de áreas verdes no limite sul - sudeste do município;

O produto gerado pode contribuir como base de dados de trabalhos futuros, assim como, auxiliar em tomadas de decisão no âmbito dos movimentos de massa no município de Belo Horizonte.

\section{Agradecimentos}

Ao Professor Diego Macedo na orientação do trabalho e a Defesa Civil de Belo Horizonte pela concessão do levantamento de ocorrência de movimentos de massa no município de 2012-2016.

\section{Referências Bibliográficas}

GUIMARÃES, F. R. Um novo olhar sobre o objeto da pesquisa em face da abordagem interdisciplinar. In: FERNANDES, A.; GUIMARÃES, F. R.; BRASILEIRO, M. E. (Org.). O fio que une a pedras: a pesquisa interdisciplinar na pós-graduação.São Paulo: Biruta, 2000. p.13-25.

PARIZZI, M. SOBREIRA. F. GALVÃO T. ARANHA. P. ELMIRO. M. BEIRIGO. E. Processos de movimentos de massa em Belo Horizonte, MG. Revistas Geografias. Belo Horizonte, 2011.

VARGAS. L. Suscetibilidade a movimentos de massa; um estudo geomorfológico na sub-bacia hidrográfica no Rio Vacacaímirim a montante da barragem do dnos, Santa Maria/RS.2015. 132p. Dissertação (Mestrado em Geografia) - Universidade Federal de Santa Maria . Rio Grande do Sul.

WICANDER, R.; JAMES, M. Fundamentos de geologia.Instituto de Geoinformação e Tecnologia IGETEC.Infraestrutura de Dados Espaciais de Minas Gerais - IEDE. Disponível em:〈http://www.iga.mg.gov.br/IEDE>Acesso em 10/01/2017.

BRASIL. Instituto Brasileiro de Geografia e Estatística - IBGE. Belo Horizonte. 2010. Disponível em: $<$ http://cidades.ibge.gov.br/xtras/perfil.php?codmun=310620>Acesso em 08/01/2017.

BELO HORIZONTE (município). Prefeitura de Belo Horizonte. Estatísticas e Indicadores - Aspectos físicos Disponível 


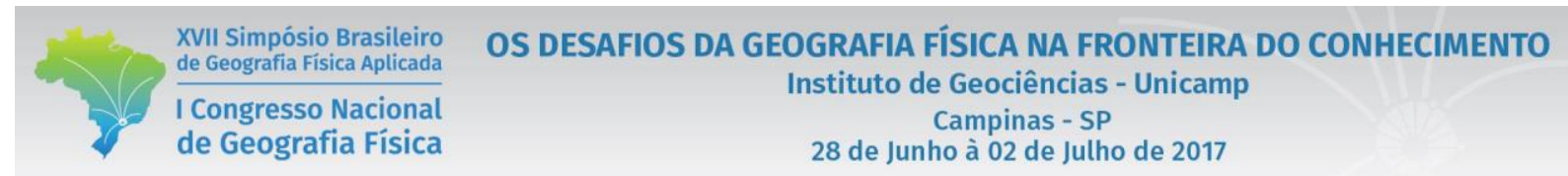

<http://portalpbh.pbh.gov.br/pbh/ecp/comunidade.do?evento=portlet\&pIdPlc=ecpTaxonomiaMenuPortal\&app=estat isticaseindicadores\&lang=pt_BR\&pg=7742\&tax=20465> Acesso em 10/01/2017.

BELO HORIZONTE (município). Gabinete Militar do Governador e Coordenadoria Estadual de Defesa Civil. Ocorrência dos movimentos de massa no município de Belo Horizonte 2012 a 2016. Belo Horizonte. 2016. 